1292

\section{A REVIEW OF INFANTILE DEATHS IN THE CHILDREN EMERGENCY, SINGAPORE}

\section{S.L. Chong}

\section{Kandang Kerbau Hospital, Singapore, Singapore}

Infantile deaths have a large impact on the family and the community.

Sudden Infant Death Syndrome has been well described.

It was proposed that Sudden Infant Death Syndrome only occurred in the presence of a lethal triad: A vulnerable infant, a critical developmental period in homeostatic control, and an exogenous stressor. Prone placement, bed-sharing and maternal smoking have been identified as risk factors. These findings have led to recommendations regarding the sleep practices among the infant population. In this study, we aim to conduct a retrospective study of all local infant deaths since 1997 to 2009. There were 53 infants aged less than 1 year who presented to the Children Emergency in cardiopulmonary collapse. Of these, $9(17.0 \%)$ were neonates, 10 $(18.9 \%)$ were $1-3$ months old and the remainder $34(64.1 \%)$ were older than 3 months. Among those deaths attributed to medical causes, 32 $(68.1 \%)$ were due to respiratory causes, 7 (14.9\%) cardiovascular causes and 2 (4.2\%) neurological causes. Only $3(6.4 \%)$ were attributed to SIDS. Among the respiratory cases, 30 (93.8\%) were due to pneumonia and 2 cases due to aspiration (6.2\%). 2 cases had positive microbiological diagnosis. Of the 4 cases attributed to trauma, 3 died from suffocation and 1 from drowning. There was 1 case of poisoning from salicylic acid.

This retrospective review serves as a basis for a future prospective study. Determination of risk factors can lead to recommendations to the community that further decrease infant death rates.

\section{3}

\section{FREQUENCY OF SERIOUS ILLNESS DURING 5-YEAR PERIOD IN OFFICE SETTINGS}

\section{N. Pejovic Mandic}

\section{Private Pediatric Practice Doktorica Mica, Podgorica, Montenegro}

Introduction: When evaluating an ill child pediatritian must be aware of statistics about the occurence of serious illness, because one of the major goals of the sick child visit is to identify the seriosly ill child. Serious illness is condition which requires the most vigorous therapeutic intervention. Asthma attack belongs to the conditions where prompt therapy is indicated, when it is moderate to severe and the main inclusion criteria is cortosteroid therapy. Serious bacterial infection was defined as pneumonia, sepsis, meningitis, pyelonephritis and osteomyelitis.

Methods: We enrolled all sick children reported for pediatric examination,0-14 years old during 5 year period,2005-2009. Retrospective analysis is done. Distribution of sick children was made according to final diagnosis and age. Age groups are $0-6$ months, 6 - 36 and more than 36 months.

Results: We analysed 10319 sick children during 5 year period. The total number of seriosly ill patients was $99(0,96 \%)$. The most frequent illness was acute asthma or bronchiolitis-42(42,42).17 (40\%) was 6 - 36 monhs aged and $23(54,00 \%)$ older than 36 months.Serious bacterial illness was found in $50(50,50 \%)$ children.29 children $(29,29 \%)$ had pyelonephritis.12(41,37\%)was in 6 - 36 months old group and $9(31,03 \%)$ were older than 36 months. 18 patients $(18,18 \%)$ had pneumonia,8 $(44,44 \%)$ in 6-36 months group and8(44,44\%) in more than 36 months group. We had only three patients with septicaemia..

Conclusion: Serious illness is very rare in our office based settings which makes it even more difficult to distinct "really sick“ children. Only watchfull follow up and rational diagnostic and therapeutic approach could lead to right decision making.

\section{4}

\section{IRON DEFICIENCY ANEMIA IN A CHILD WITH IDIOPATHIC PULMONARY HEMOSIDEROSIS}

S. Corujeira1 , R. Santos-Silva ${ }^{1}$, F. Ferreira ${ }^{2}$, C. Rego ${ }^{1}$, A. Maia ${ }^{1}$, I. Azevedo ${ }^{1}$

${ }^{1}$ Paediatrics, ${ }^{2}$ Haematology, Hospital São João, Porto, Portugal

Background: Idiopathic pulmonary hemosiderosis $(\mathrm{IPH})$ is a rare disease with a variable outcome. It is characterized by recurrent episodes of diffuse alveolar hemorrhage and iron deficiency anemia.

Case Report: A 6-year-old boy, was hospitalized for severe anemia. At the time of the diagnosis he presented with progressive fatigue and pallor. The physical examination revealed mucocutaneous 
pallor, a grade III/VI systolic murmur and inspiratory crackles. Laboratory investigation revealed microcytic hypochromic anemia (hemoglobin $5.7 \mathrm{~g} / \mathrm{dL}$, mean corpuscular volume of $58.9 \mathrm{fL}$, reticulocyte index 2.18) with iron deficiency, normal renal and liver function, and normal coagulation profile. Admitted under iron suplementation with progressive rise in hemoglobin. Infectious causes were excluded. Bone marrow biopsy was normal. After 1 month he developed fever, cough and dispnoea, without hemoptysis. The chest radiograph showed diffuse alveolar-type infiltrates. Bronchoscopy with bronchoalveolar lavage revelead over $75 \%$ haemosiderin -stained macrophages. Lung biopsy specimen was negative for vasculitis, immune complex deposition or granulomatous disease. The immunological study was negative, including anti-GBM, ANA, c-ANCA, IgE cow's milk antibodies and reumatoid factor. Echocardiography revealed no evidence of pulmonary hypertension. The diagnosis of IPH was considered and the patient was started on prednisolone $(2 \mathrm{mg} / \mathrm{kg} / \mathrm{day}$ ) with improvement in respiratory symptoms after 1 week. Despite corticosteroids he kept having IPH exacerbations and chronic anemia and initiated hydroxichloroquine with good clinical response.

Comments: In this case the association of iron deficiency anemia refractory to iron supplementation with the sudden onset of respiratory symptoms and the suggestive radiological presentation established the diagnosis of IPH.

\section{5}

DRUG ADMINISTRATION RELATED PROBLEMS IN OUTPATIENT CHILDREN: MORE THAN FORMULATION ASPECTS

D. van Riet-Nales ${ }^{1}$, N. Bouwman ${ }^{2}$, A.F.A.M. Schobben' ${ }^{2}$, T.C.G. Egberts ${ }^{2,3}$, R. Carin ${ }^{3}$

${ }^{1} R I V M$, Center for the Quality of Chemical Pharmaceutical Products (KCF), ${ }^{2}$ Division of Epidemiology and Pharmacotherapy, Utrecht Institute for Pharmaceutical Sciences, Utrecht University, ${ }^{3}$ University Medical Center Utrecht, Utrecht, The Netherlands

Background: Optimal pharmacotherapy for children requires that drugs can be easily administered. However, parents often encounter problems leading to medication errors. The aim of this study was to explore the nature of drug administration related problems in outpatient children and to assign their most likely solutions.
Methods: A questionnaire was issued to parents of outpatient children (0-12 years) and adolescents (1218 years). Complaints relating to the administration of tablets, capsules, oral liquid preparations, rectal preparations or preparations for throat, nose ear or eye were identified, categorized and compiled into a patient related or product related problem. The most likely solutions to these problems were assigned.

Results: One hundred questionnaires were returned, 71 of them reporting 154 different complaints (including multiple complaints about the same product). These complaints were compiled into 68 patient related and 20 product related problems. Twenty-eight (41\%) of the patient related problems were most likely to be solved by product optimization, $12(18 \%)$ by behavioural interventions and $28(41 \%)$ by a combination of both. Seventeen $(85 \%)$ of the product related problems were most likely to be solved by product optimization, 2 (10\%) by behavioural interventions and $1(5 \%)$ by a combination of both.

Conclusion: Interventions to address problems in paediatric drug administration cannot be limited to formulation aspects. In order to make medicines more acceptable to children a broader approach is required involving both product optimization as well as guidance for improvement of behavioural aspects.

\section{6}

\section{FELT OR ENACTED CRITICISM THEORY - A CONTRIBUTION TO THE UNDERSTANDING OF PARENTS' DECISION MAKING IN ACUTE CHILDHOOD ILLNESS}

\author{
S. Neill ${ }^{1}$, S. Cowley ${ }^{2}$
}

${ }^{1}$ School of Health, University of Northampton, Northampton, ${ }^{2}$ Florence Nightingale School of Nursing \& Midwifery, King's College London, London, UK

Acute childhood illness is the most common experience of illness in families. These illnesses create considerable uncertainty and anxiety for parents concerning when to seek help for a sick child. This paper explores findings, from a doctoral research project, which contribute to understanding of parents' decision making at these times. This grounded theory study used 24 in-depth interviews in 4 rounds of data collection to explore psychosocial processes in 15 families when children are acutely 